in vivo $33: 1499-1505$ (2019)

doi:10.21873/invivo.11630

\title{
The Role of Fgf Signaling on Epithelial Cell Differentiation in Mouse Vagina
}

\author{
YU HIRANO ${ }^{1}$, KENTARO SUZUKI $^{1}$, TAISEN IGUCHI ${ }^{2}$, GEN YAMADA $^{1}$ and SHINICHI MIYAGAWA ${ }^{3}$ \\ ${ }^{1}$ Department of Developmental Genetics, Institute of Advanced Medicine, \\ Wakayama Medical University, Wakayama, Japan; \\ ${ }^{2}$ Graduate School of Nanobioscience, Yokohama City University, Yokohama, Japan; \\ ${ }^{3}$ Faculty of Industrial Science and Technology, Tokyo University of Science, Tokyo, Japan
}

\begin{abstract}
Background/Aim: The mouse vagina exhibits stratified squamous epithelium, which is comprised of multiple cell layers. We previously showed that erbB signaling, induced by epithelial estrogen receptor 1 (ESRI), is required for the initial differentiation of the epithelium. However, the downstream effector that mediates terminal differentiation in the apical layers remains elusive. The contribution of fibroblast growth factor ( $F G F)$ to vaginal epithelial cell differentiation was investigated. Materials and Methods: Vaginas from wild-type or epithelium-specific Esr I conditional knockout (cKO) mice were analyzed using immunohistochemistry and quantitative real-time RT-PCR. Results: Of the FGF ligands examined, Fgf22 mRNA was significantly induced following estrogen treatment. Furthermore, FGF downstream signaling, phosphorylated FRS2 and ERK1/2 were exclusively expressed in the apical layers of the vaginal epithelium. No changes in such expression were observed in the Esr 1 cKO mice. Conclusion: FGF-ERK/MAPK pathway may be a main inducer of terminal differentiation in the mouse vaginal epithelium.
\end{abstract}

Estrogens play important roles in regulating mammalian organ homeostasis. In particular, cell proliferation and differentiation of female reproductive organs, such as

This article is freely accessible online.

Correspondence to: Shinichi Miyagawa, Department of Biological Science and Technology, Faculty of Industrial Science and Technology, Tokyo University of Science, Niijuku 6-3-1, Katsushika, Tokyo 125-8585, Japan. Tel: +81 358761466, Fax: +81 358761466, e-mail: miyagawa@rs.tus.ac.jp and Gen Yamada, Institute of Advanced Medicine, Wakayama Medical University, Kimiidera 811-1, Wakayama, Wakayama 641-8509, Japan. Tel: +81 734995026, Fax: +81 734995026, e-mail: genyama77@yahoo.co.jp

Key Words: Estrogen receptor, vagina, keratinization, fibroblast growth factors, epithelium. mammary gland, uterus and vagina, are tightly regulated by estrogens (1). In vagina, estrogens induce epithelial cell proliferation in the basal layer (2). The suprabasal cells are no longer mitogenic and differentiate while moving up through the epithelium (2). The apical cells exhibit keratinization, and they lose their nuclei and cytoplasm, which are eventually shed from the surface (2). The fully stratified and keratinized epithelium in the vagina resembles epidermis in the skin; however, unique and comprehensive process of this epithelial cell differentiation in mouse vagina, which is again regulated by estrogens, remains elusive.

Estrogens activate their target gene expression mainly through binding to the nuclear estrogen receptors $\alpha$ and $\beta$ (ESR1 and ESR2) (1). Classical tissue recombination experiments have shown that epithelial cell proliferation is mediated through stromal ESR1, whereas epithelial cell differentiation requires epithelial ESR1 (3-5). Our in vivo study with vaginal epithelium-specific Esr $1 \mathrm{cKO}$ mice has also revealed that epithelial ESR1 is required for cell differentiation; the vagina of cKO mice did not express cytokeratin 1 (CK1) and filaggrin, early and terminal differentiation maker proteins, respectively (6). We also found that amphiregulin (Areg) is a mediator for CK1 expression in mouse vagina in response to estrogen. Areg is, however, insufficient for full expression of filaggrin and terminal differentiation (6). Therefore, additional downstream factors are necessary for full activation of estrogen effects and terminal differentiation in the epithelium of the mouse vagina.

Fibroblast growth factor (FGF) plays crucial roles for embryonic development and tissue homeostasis by regulating cell proliferation, migration and differentiation (7). The FGF family is composed of 22 secreted proteins, which are divided into 7 subfamilies (7). They interact with tyrosine kinase FGF receptors (FGFRs). Binding of FGFs facilitates dimerization of FGFRs and intracellular conformational changes, which activate tyrosine kinase domains and phosphorylates intermediate docking proteins, such as FGFR substrate 2 (FRS2) (8-11). The phosphorylated FRS2 (pFRS2) recruits the 
Primer sequences $\left(5^{\prime}-3^{\prime}\right)$

\begin{tabular}{lll}
\cline { 2 - 3 } Gene & Forward & Reverse \\
\hline$L 8$ & ACAGAGCCGTTGTTGGTGTTG & CAGCAGTTCCTCTTTGCCTTGT \\
$F g f 3$ & ACGGCAGCCTTAAGAACA & CCACTTCCACCGCAGTAATC \\
$F g f 7$ & AAGGGACCCAGGAGATGAAG & ACTGCCACGGTCCTGATTT \\
$F g f 8$ & CATGGCAGAAGACGGAGAC & ACTCGGACTCTGCTTCCAAA \\
$F g f 10$ & GCGGGACCAAGAATGAAGA & AGTTGCTGTTGATGGCTTTGA \\
$F g f 18$ & GGCAAATCCGTGAATACCA & CTGCTGCCGAATGTATCTGT \\
$F g f 22$ & TGCTGTGCTTCCAGGTTCA & GGATGCGGAAGTCCACATT \\
\hline
\end{tabular}

adaptor protein, growth factor receptor-bound 2 (GRB2), and the exchange factor, son of sevenless (SOS) (8-11). As a result, SOS activates the RAS-GTPase, which induces activation of the mitogen-activated protein kinase (MAPK) pathway, including the phosphorylation of extracellular signalregulated kinases 1 and 2 (pERK1/2) (8-11). During the mouse vaginal development, the FGF-ERK/MAPK pathway is one of the key mediators of lineage determination of the neonatal Müllerian duct epithelium to the vaginal epithelium (12-14); however, the contribution of FGF signaling to the homeostasis of adult vaginal epithelium is still unclear.

In this study, we focused on the FGF signaling in order to understand the molecular pathway of the estrogen-mediated cell differentiation in the adult mouse vagina. Our results suggest that the activation of FGF-ERK/MAPK pathway is involved in a series of ESR1-mediating events, particularly in the terminal differentiation of the vaginal epithelium.

\section{Materials and Methods}

Mouse and treatment. All experimental procedures and protocols were approved by the Animal Research Committee of the Wakayama Medical University (Approval Number: 28-2 and 28-9). The mouse strains used were C57BL/6J (CLEA, Tokyo, Japan), K5-Cre (15), Esr1-null and Esr1-floxed (16). All lines were maintained in the C57BL/6J background. K5-Cre mice and Esr 1 -null mice were crossed to obtain male $\mathrm{K} 5$-Cre/+; $\mathrm{Esrl}^{+/-}$mice. Esr1 cKO mice (K5-Cre/+; Esrlflox/-) were obtained by crossing $\mathrm{K} 5-\mathrm{Cre} /+$; $\mathrm{Esrl}^{+/-}$male mice with Esr Iflox/flox female mice. For control mice, $\mathrm{K} 5 \mathrm{Cre} /-;$ Esr Iflox/+ littermates were used. Mice were ovariectomized (OVX) at 6 weeks of age and were culled at 8 weeks of age. To test the effects of estrogen, a single daily injection of $0.1 \mu \mathrm{g} 17 \beta$-estradiol $\left(\mathrm{E}_{2}\right.$, Sigma, St Louis, MO, USA) dissolved in sesame oil (Kanto Chemical, Tokyo, Japan) was given to OVX mice for 3 days and sacrificed 24 hours following the last injection. Unless otherwise mentioned, materials were purchased from Wako Pure Chemical, Osaka, Japan.

Immunohistochemistry. Eight weeks mice vaginas were fixed overnight in $4 \%$ paraformaldehyde (Sigma) with phosphate-buffered saline (PBS), embedded in paraffin, and sectioned on $8 \mu \mathrm{m}$. Antigen retrieval was performed by incubating with $0.1 \mathrm{mM}$ citrate buffer $(\mathrm{pH} 6.0)$ in a microwave $(200 \mathrm{~W})$ for $10 \mathrm{~min}$. Endogenous peroxidase activity was inactivated in methanol containing $0.3 \%$ $\mathrm{H}_{2} \mathrm{O}_{2}$ for 30 minutes. Following 1 hour incubation with $1.5 \%$ fetal bovine serum (FBS, Thermo Fisher Scientific, Waltham, MA, USA) in PBS as a blocking buffer, the following primary antibodies were used on the slides: anti-pERK1/2 (1:200, Cell Signaling, Danvers, MA, USA), anti-pFRS2 (1:200, R\&D Systems, Minneapolis, MN, USA) and anti-pAKT (1:200, D9E, Cell Signaling). The sections were incubated with the primary antibody diluted in blocking buffer at $4^{\circ} \mathrm{C}$ for overnight. Final visualization was performed with the Vectastain ABC Kit (Vector Laboratories, Burlingame, CA, USA).

Quantitative real-time $R T-P C R$ ( $q R T-P C R)$. Gene expression changes were quantified using the StepOnePlus Real-Time PCR System (Thermo Fisher Scientific), according to the manufacturer's instructions. Total RNA ( $2 \mu \mathrm{g})$ was isolated from whole mouse vaginas at 8 weeks of age using an Isogen RNA extraction kit (Nippongene, Toyama, Japan). Synthesis of cDNA was performed with SuperScript III reverse transcriptase (Thermo Fisher Scientific) and the resulting cDNA was mixed with the SYBR premix Ex Taq (Takara, Ohtsu, Japan). Three pools of samples per group were assayed in triplicates, and three samples were assayed. The relative RNA equivalents for each sample were determined by a comparison to the levels of the normalized standard ribosomal protein L8 (L8), and then calculated as the fold change compared to the normalized standard. The sequences of gene primers are shown in Table I (17, 18). Statistical analysis was performed by Tukey-Kramer test followed by two-way ANOVA. Differences with $p<0.05$ were considered significant. Error bars represent the standard error of the mean (SEM).

\section{Results}

Expression patterns of Fgf ligands in the adult vagina. The signaling mechanism mediated through secreted growth factors, enables the amplification and systematic coordination of estrogen-induced epithelial cell proliferation and differentiation in the female reproductive organs. In this study, we focused on the FGF signaling, one of the major families of secreted growth factors. We have previously 

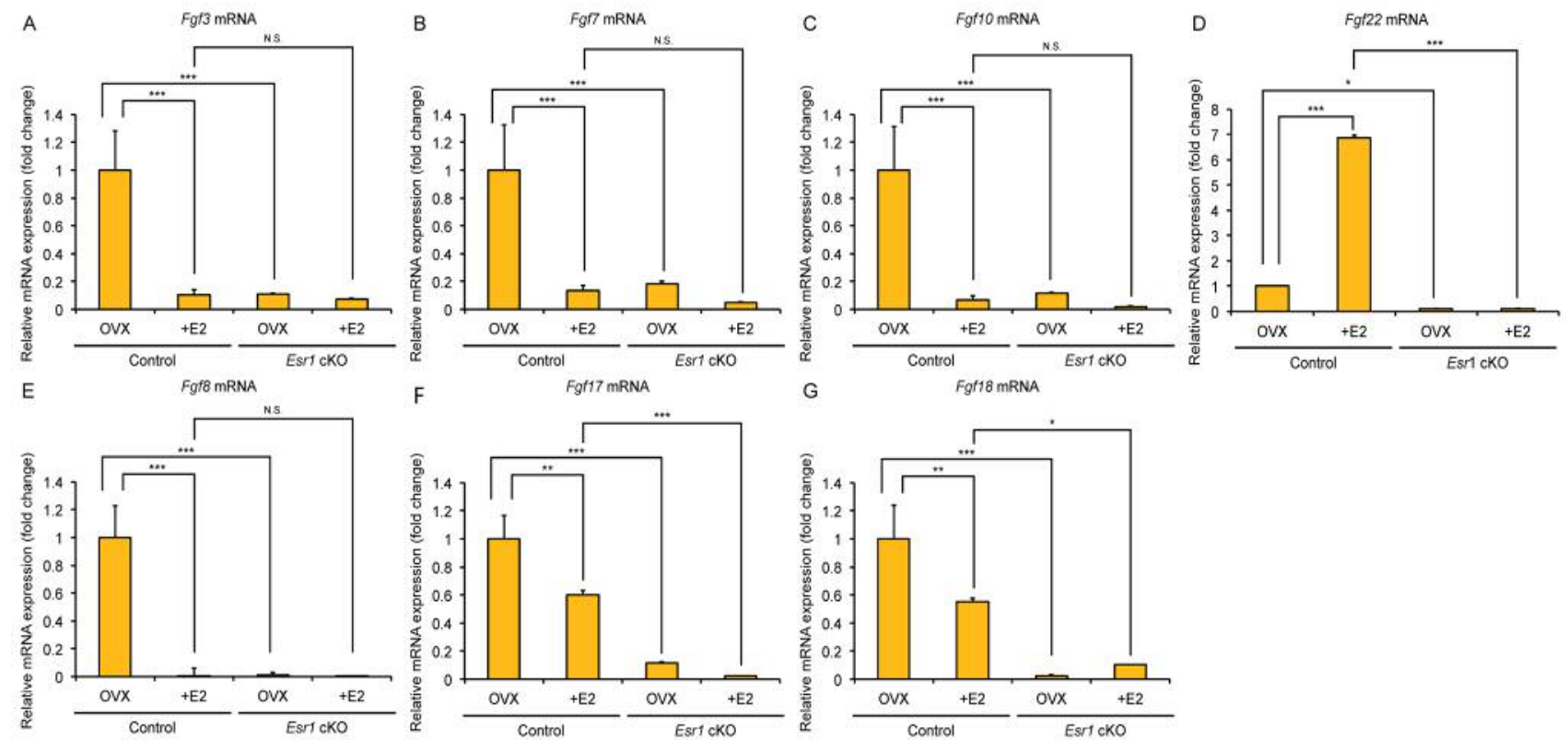

Figure 1. Expression profile of FGF ligands in control and Esr1 cKO mouse vagina. Expression of Fgf3 (A), Fgf7 (B), Fgf10 (C), Fgf22 (D), Fgf8 $(E), F g f 17(F), F g f 18(G)$ in mouse vagina are measured with quantitative real-time RT-PCR. Of these ligands, Fgf22 mRNA is significantly induced following $E_{2}$ treatment in the control mouse. $\left({ }^{*} p<0.05\right.$, ${ }^{*} p<0.01$, ${ }^{* *} p<0.001$, N.S.: Not significant $)(n=3)$.

found that $F g f 22$ and $F g f 18$ expression are significantly decreased in the vagina of $E s r l \mathrm{cKO}$ mice, which display failure of keratinized differentiation, suggesting that these FGF members are possibly involved in the epithelial cell differentiation (6). We first performed quantitative real-time RT-PCR (qRT-PCR) analysis for several FGF ligands expression in the Esr 1 cKO mouse vagina. To remove any confounding biases of the hypothalamic-pituitary-gonadal axis and to simplify the analysis of estrogen effects, mice were ovariectomized and treated with $17 \beta$-estradiol $\left(\mathrm{E}_{2}\right)$ for 3 days, which provides sufficient time for inducing stratified and adequately keratinized epithelium in the mouse vagina $(6,19)$. We tested several $F g f$ ligands: i) $F g f 22$, ii) $F g f 3$, iii) $F g f 7$, iv) Fgflo (FGF7 subfamily), v) Fgfl8, vi) Fgf8, vii) Fgf17 (FGF8 subfamily). In the control mice, $\mathrm{E}_{2}$-treatment led to the downregulation of most $F g f s$ mRNA expression, except for $F g f 22$, which was significantly augmented upon $\mathrm{E}_{2}$ stimulation (Figure 1 and Table II). In the Esrl cKO mice, such upregulation was not observed even following $E_{2}$ treatment.

FGF-MAPK signals were detected in the apical layer. We next examined the phosphorylation of FRS2 and ERK1/2, presumptive downstream targets of $\mathrm{Fgf}$ signaling. The vaginal epithelia of control and Esrl cKO ovariectomized (OVX) mice were form two or three cell layers (Figure 2A and $\mathrm{B})$. In control mice, $\mathrm{E}_{2}$ treatment induced epithelial stratification and keratinization, and pFRS2 was detected in the apical layer of the vaginal epithelium (Figure 2C). By contrast, vaginal epithelium of Esrl cKO mice failed in keratinization and did not express pFRS2 (Figure 2D). Similar to pFRS, pERK1/2 expression was detected in the apical layers of epithelium in $\mathrm{E}_{2}$-treated control vaginas, but not in the Esrl cKO vaginas (Figure 2E, F, G and H).

It has been reported that FGF and erbB signaling activate other cellular signal transduction pathway, such as phosphatidylinositol 3-kinase (PI3K) and AKT (20-26). Phosphorylated-AKT (pAKT) was expressed in the suprabasal (middle) layer rather than in apical layers in response to estrogen (Figure 2I, J, K and L), suggesting that $\mathrm{PI} 3 \mathrm{~K} / \mathrm{AKT}$ signaling is not a main inducer of terminal differentiation in mouse vagina. These data also suggest that FGF-ERK/MAPK pathway activated by ESR 1 mainly regulates the vaginal epithelial terminal differentiation.

\section{Discussion}

Estrogen tightly regulates cell proliferation and differentiation in the female reproductive organs. Previous tissue recombination experiments and conditional knockout mice studies have revealed that these events are mediated through complicated tissue-tissue interactions $(3-6,19)$. As in the case of many stratified epithelia, vaginal epithelium is maintained through the proliferation of basal cells, which requires stromal 


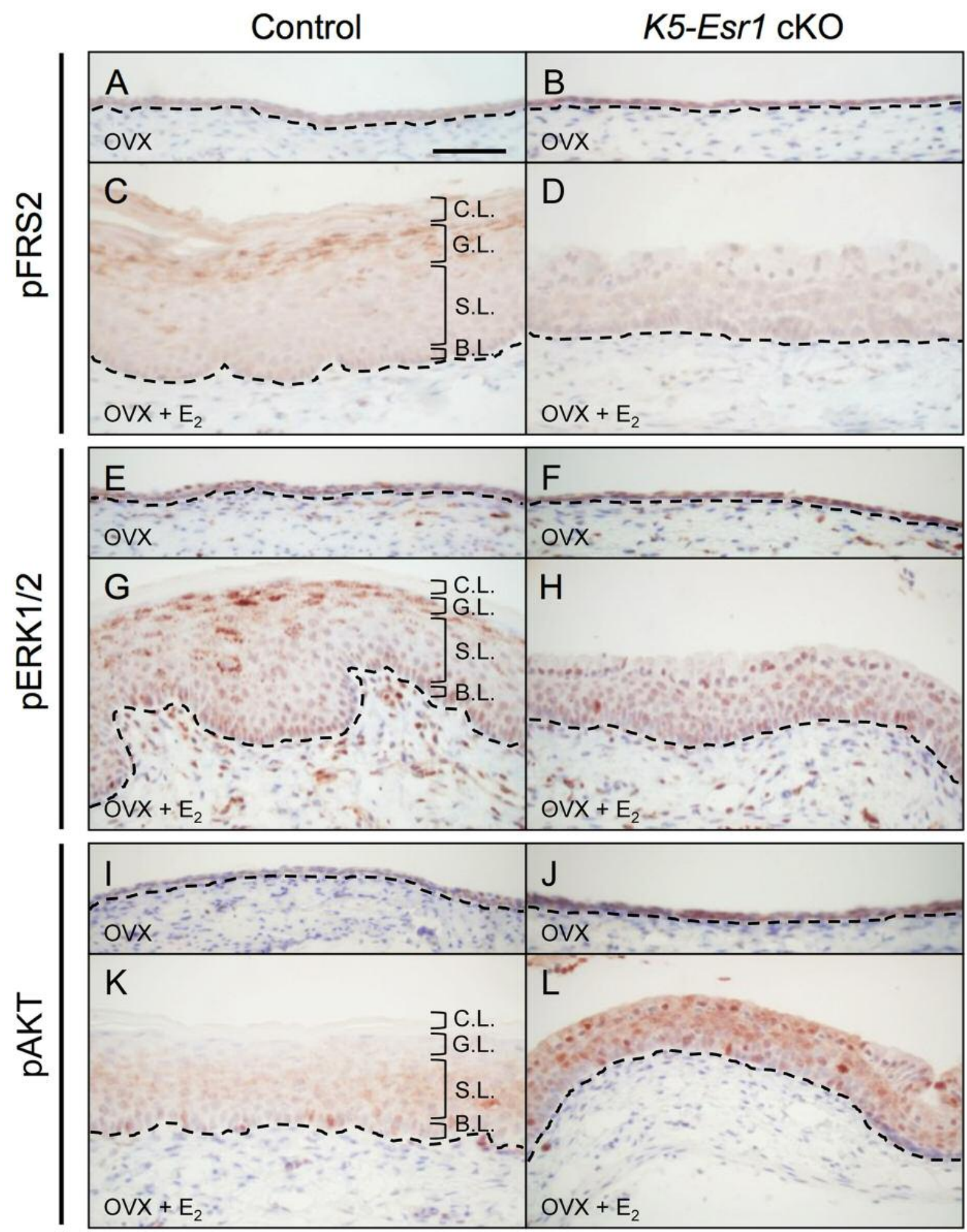

Figure 2. Expression pattern of FGF-MAPK and AKT signaling proteins in control and Esr1 cKO mouse vagina. The vaginal epithelia from OVX control mice show two or three cell layers $(A, B, E, F, I$ and $J)$. Expression of $p F R S 2$ and $p E R K 1 / 2$ are detected in $E_{2}$-treated vaginal epithelia of control mice, particularly in the apical layer $(C$ and $G)$. The vaginal epithelia from Esr1 cKO mice show failure of cell differentiation, stratification and phosphorylation of pFRS2 and pERK1/2 $(D$ and $H)$. pAKT is expressed in the middle layer of $E_{2}$-treated vaginal epithelia of control mice and is augmented in the whole epithelium of $E_{2}$-treated Esrl cKO vagina $(K$ and $L)$. Dotted lines indicate the basal layer of the epithelium. Scale bar=100 um. (n=3). B.L.: Basal Layer; S.L.: Spinous Layer (Suprabasal Layer); G.L.: Granular Layer (Apical Layer); C.L.: Cornified Layer. 
Table II. The results of qRT-PCR for Fgf ligands.

\begin{tabular}{|c|c|c|c|c|}
\hline \multirow[b]{2}{*}{ Subfamily } & \multirow[b]{2}{*}{ Gene } & \multicolumn{3}{|c|}{$p$-Value } \\
\hline & & Control-OVX vs. Control- $\mathrm{E}_{2}$ & Control-OVX vs. Esr1 cKO-OVX & Control- $\mathrm{E}_{2}$ vs. Esr1 cKO- $\mathrm{E}_{2}$ \\
\hline \multirow[t]{4}{*}{ FGF7 subfamily } & $F g f 3$ & $<0.001 * * *$ & $<0.001 * * *$ & 0.859 \\
\hline & $F g f 7$ & $<0.001 * * *$ & $<0.001 * * *$ & 0.168 \\
\hline & Fgflo & $<0.001 * * *$ & $<0.001 * * *$ & 0.454 \\
\hline & $F g f 22$ & $<0.001 * * *$ & $0.034^{*}$ & $<0.001 * * *$ \\
\hline \multirow[t]{3}{*}{ FGF8 subfamily } & $F g f 8$ & $<0.001 * * *$ & $<0.001 * * *$ & 0.954 \\
\hline & Fgf17 & $0.006^{* *}$ & $<0.001 * * *$ & $<0.001 * * *$ \\
\hline & Fgfl8 & $0.007 * *$ & $<0.001 * * *$ & $0.013^{*}$ \\
\hline
\end{tabular}

$* p<0.05, * * p<0.01, * * * p<0.001$

ESR1 expression and secretion of the proliferative factors (3$5)$. By contrast, epithelial cell differentiation is directly mediated by epithelial ESR1 (6). Suprabasal cells exit the cell cycle and enter a squamous cell differentiation processes, however, in the absence of epithelial ESR1, keratinized differentiation fails (6). In the mouse vaginal epithelium, interactions among multiple cell layers coordinated through various secretory signaling factors regulate epithelial cell homeostasis $(6,19)$. This is essential for the amplification of estrogen stimulation in the female reproductive organs. We have found that the erbB ligand, AREG, is expressed specifically in the epithelium, and is dependent on epithelial ESR1 expression $(6,17)$. Organ culture experiments have revealed that AREG compensates for the loss of epithelial ESR1 and induces CK1 expression, an early cell differentiation marker, even in the absence of estrogens. Nevertheless, AREG is insufficient for the full expression of filaggrin and terminal differentiation (6). Thus, other factors are likely to be involved in further epithelial cell differentiation.

In this study, we found that FGF signaling is a possible candidate involved in the terminal differentiation in mouse vagina. Of the Fgf ligands examined, $F g f 22$ was unique to be induced following $\mathrm{E}_{2}$ treatment in the control mouse vagina. FGF22 is classified into the same group as FGF7 (7), and is called keratinocyte growth factor (KGF), regulating the cell differentiation and regeneration of various epithelial tissues, such as esophagus, lung, trachea and skin (27-32). KGF treatment in the neonatal or adult mouse vagina results in fully stratified and keratinized epithelium, suggesting a relationship between estrogen signaling and activation of FGF signaling (33-35). KGF and FGF22 bind to FGFR2IIIb $(36,37)$, which is predominantly expressed in epithelial cells, and is essential for epithelial cell proliferation, differentiation and homeostasis (38-40). From these data, we hypothesized that $F g f 22$ is involved in the terminal differentiation of the mouse vagina as a downstream factor of estrogens, although it is unclear whether ESR1 directly regulates Fgf22 expression. By contrast, Fgf18 was not induced following $\mathrm{E}_{2}$ treatment in the current study. In the mouse uterus, it has been reported that FGF18 is induced by stromal ESR1 and possibly mediates epithelial cell proliferation $(41,42)$. Thus, we cannot rule out a possible role of FGF18 on the vaginal epithelial cell homeostasis. Further studies are needed in order to elucidate the regulatory mechanisms of FGF signaling by estrogens in the female reproductive tracts with a clear view of the differences between uterus and vagina.

pFRS2 and pERK1/2 were co-expressed in the apical layers of mouse vagina, suggesting that the FGF-ERK/MAPK pathway can be a main inducer of terminal differentiation. By contrast, pAKT was detected in the suprabasal layers of the mouse vagina, where early differentiating cells are localized. Thus, the PI3K/AKT pathway is possibly activated by erbB signaling in the mouse vagina (22-26). Akt phosphorylated by PI3K can lead to the stabilization of the cell cycle inhibitor, p21 (43). It is, thus, suggested that PI3K/AKT might promote cell differentiation by attenuating cell proliferation in the mouse vaginal epithelium. In fact, p21 is expressed in the suprabasal cells of the mouse vaginal epithelium upon estrogen treatment (44). Intriguingly, pAKT was found to be augmented in the whole epithelial layers of $\mathrm{E}_{2}$-treated Esrl cKO vaginas. In these mice, the suprabasal layers of the epithelium have been shown to exhibit aberrant and ectopic cell proliferation, which never happens in the normal stratified epithelium (6). Considering the results, this augmented pAKT promotes ectopic cell proliferation in the whole epithelium of Esrl cKO vagina.

Taken together, our results suggest that FGF-ERK/MAPK signaling is involved in the vaginal epithelial terminal differentiation. Overall, we unraveled the mechanism of estrogen signaling in the mouse vagina, which is key to the elucidation of estrogen-mediated events in the female reproductive organs, including disorders such as hormoneinduced tumorigenesis. 


\section{Conflicts of Interest}

None of the Authors has any potential conflicts of interest associated with this research.

\section{Authors' Contribution}

Y.H., T.I. and S.M. designed the research. Y.H. and T.I. collected tissue samples and performed experiments. Y.H., K.S. and G.Y. analyzed data. Y.H. and S.M. supervised the research and wrote the manuscript.

\section{Acknowledgements}

The Authors thank Drs. P. Chambon and J. Takeda for providing Esr1-floxed and K5-Cre mice respectively, and Drs. D. Matsumaru, A.Murashima, D. Kajioka, R.Yatsu, K. Toyota for their intellectual input. They would also like to thank Mr. H. Saeki and Ms. T.I. Iba for their assistance. This work was supported by Japan Society for the Promotion of Science KAKENHI Grant Number 17H06432, 18H02474, 18K06837, 18K06938.

\section{References}

1 Couse JF and Korach KS: Estrogen receptor null mice: what have we learned and where will they lead us? Endocr Rev 20(3): 358-417, 1999. PMID: 10368776. DOI: 10.1210/edrv.20.3.0370

2 Cunha GR, Lee AK and Lung B: Electron microscopic observations of vaginal development in untreated and neonatally estrogenized Balb/c Crgl mice. Am J Anat 152(3): 343-81, 1978. PMID: 677055. DOI: 10.1002/aja.1001520307

3 Buchanan DL, Kurita T, Taylor JA, Lubahn DB, Cunha GR and Cooke PS: Role of stromal and epithelial estrogen receptors in vaginal epithelial proliferation, stratification, and cornification. Endocrinology 139(10): 4345-52, 1998. PMID: 9751518. DOI: 10.1210/endo.139.10.6241

4 Buchanan DL, Setiawan T, Lubahn DB, Taylor JA, Kurita T, Cunha GR and Cooke PS: Tissue compartment-specific estrogen receptor-alpha participation in the mouse uterine epithelial secretory response. Endocrinology 140(1): 484-91, 1999. PMID: 9886861. DOI: $10.1210 /$ endo.140.1.6448

5 Kurita T, Cooke PS and Cunha GR: Epithelial-stromal tissue interaction in paramesonephric (Müllerian) epithelial differentiation. Dev Biol 240(1): 194-211, 2001. PMID: 11784056. DOI: 10.1006/ dbio.2001.0458

6 Miyagawa $\mathrm{S}$ and Iguchi T: Epithelial estrogen receptor 1 intrinsically mediates squamous differentiation in the mouse vagina. Proc Natl Acad Sci USA 112(42): 12986-91, 2015. PMID: 26438838. DOI: 10.1073/pnas.1513550112

7 Ornitz DM and Itoh N: The Fibroblast Growth Factor signaling pathway. Wiley Interdiscip Rev Dev Biol 4(3): 215-66, 2015. PMID: 25772309. DOI: 10.1002/wdev.176

8 Kouhara H, Hadari YR, Spivak-Kroizman T, Schilling J, BarSagi D, Lax I and Schlessinger J: A lipid-anchored Grb2-binding protein that links FGF-receptor activation to the Ras/MAPK signaling pathway. Cell 89(5): 693-702, 1997. PMID: 9182757.

9 Hadari YR1, Kouhara H, Lax I and Schlessinger J: Binding of Shp2 tyrosine phosphatase to FRS2 is essential for fibroblast growth factor-induced PC12 cell differentiation. Mol Cell Biol 18(7): 396673, 1998. PMID: 9632781. DOI: 10.1128/mcb.18. 7.3966
10 Hadari YR, Gotoh N, Kouhara H, Lax I and Schlessinger J: Critical role for the docking-protein FRS2 alpha in FGF receptor-mediated signal transduction pathways. Proc Natl Acad Sci USA 98(15): 8578-83, 2001. PMID: 11447289. DOI: 10.1073/pnas.161259898

11 Lax I, Wong A, Lamothe B, Lee A, Frost A, Hawes J and Schlessinger J: The docking protein FRS2 alpha controls a MAP kinase-mediated negative feedback mechanism for signaling by FGF receptors. Mol Cell 10(4): 709-19, 2002. PMID: 12419216.

12 Nakajima T, Hayashi S, Iguchi $\mathrm{T}$ and Sato $\mathrm{T}$ : The role of fibroblast growth factors on the differentiation of vaginal epithelium of neonatal mice. Differentiation 82(1): 28-37, 2011. PMID: 21530063. DOI: 10.1016/j.diff.2011.03.005

13 Terakawa J, Rocchi A, Serna VA, Bottinger EP, Graff JM and Kurita T: FGFR2IIIb-MAPK Activity Is Required for Epithelial Cell Fate Decision in the Lower Müllerian Duct. Mol Endocrinol 30(7): 78395, 2016. PMID: 27164167. DOI: 10.1210/me.2016-1027

14 Atsuta Y and Takahashi Y: Early formation of the Müllerian duct is regulated by sequential actions of BMP/Pax 2 and FGF/Lim1 signaling. Development 143(19): 3549-3559, 2016. PMID: 27578782. DOI: $10.1242 /$ dev.137067

15 Tarutani M, Itami S, Okabe M, Ikawa M, Tezuka T, Yoshikawa $\mathrm{K}$, Kinoshita T and Takeda J: Tissue-specific knockout of the mouse Pig-a gene reveals important roles for GPI-anchored proteins in skin development. Proc Natl Acad Sci USA 94(14): 7400-5, 1997. PMID: 9207103. DOI: 10.1073/pnas.94.14.7400

16 Dupont S, Krust A, Gansmuller A, Dierich A, Chambon P and Mark M: Effect of single and compound knockouts of estrogen receptors alpha (ERalpha) and beta (ERbeta) on mouse reproductive phenotypes. Development 127(19): 4277-91, 2000. PMID: 10976058.

17 Miyagawa S, Katsu Y, Watanabe $H$ and Iguchi T: Estrogenindependent activation of erbBs signaling and estrogen receptor alpha in the mouse vagina exposed neonatally to diethylstilbestrol. Oncogene 23(2): 340-9, 2004. PMID: 14647453. DOI: 10.1038/sj.onc.1207207

18 Fon Tacer K, Bookout AL, Ding X, Kurosu H, John GB, Wang L, Goetz R, Mohammadi M, Kuro-o M, Mangelsdorf DJ and Kliewer SA: Research resource: Comprehensive expression atlas of the fibroblast growth factor system in adult mouse. Mol Endocrinol 24(10): 2050-64, 2010. PMID: 20667984. DOI: 10.1210/me.2010-0142

19 Miyagawa S, Sato M, Sudo T, Yamada G and Iguchi T: Unique roles of estrogen-dependent Pten control in epithelial cell homeostasis of mouse vagina. Oncogene 34(8): 1035-43, 2015. PMID: 24632614. DOI: 10.1038/onc.2014.62

20 Lamothe B, Yamada M, Schaeper U, Birchmeier W, Lax I and Schlessinger $\mathrm{J}$ : The docking protein Gab1 is an essential component of an indirect mechanism for fibroblast growth factor stimulation of the phosphatidylinositol 3-kinase/Akt antiapoptotic pathway. Mol Cell Biol 24(13): 5657-66, 2004. PMID: 15199124. DOI: 10.1128/MCB.24.13.5657-5666.2004

21 Maehara O, Suda G, Natsuizaka M, Ohnishi S, Komatsu Y, Sato F, Nakai M, Sho T, Morikawa K, Ogawa K, Shimazaki T, Kimura M, Asano A, Fujimoto Y, Ohashi S, Kagawa S, Kinugasa H, Naganuma S, Whelan KA, Nakagawa H, Nakagawa K, Takeda $H$ and Sakamoto N: Fibroblast growth factor-2-mediated FGFR/Erk signaling supports maintenance of cancer stem-like cells in esophageal squamous cell carcinoma. Carcinogenesis 38(11): 1073-1083, 2017. PMID: 28927233. DOI: 10.1093/carcin/bgx095 
22 Toulany M, Baumann M and Rodemann HP: Stimulated PI3KAKT signaling mediated through ligand or radiation-induced EGFR depends indirectly, but not directly, on constitutive K-Ras activity. Mol Cancer Res 5(8): 863-72, 2007. PMID: 17699110. DOI: 10.1158/1541-7786.MCR-06-0297

23 Yotsumoto F, Fukami T, Yagi H, Funakoshi A, Yoshizato T, Kuroki $M$ and Miyamoto S: Amphiregulin regulates the activation of ERK and Akt through epidermal growth factor receptor and HER3 signals involved in the progression of pancreatic cancer. Cancer Sci 101(11): 2351-60, 2010. PMID: 20726858. DOI: $10.1111 / j .1349-7006.2010 .01671 . x$

24 Peterson EA, Jenkins EC, Lofgren KA, Chandiramani N, Liu H, Aranda E, Barnett M and Kenny PA: Amphiregulin Is a Critical Downstream Effector of Estrogen Signaling in ER $\alpha$-Positive Breast Cancer. Cancer Res 75(22): 4830-8, 2015. PMID: 26527289. DOI: 10.1158/0008-5472.CAN-15-0709

25 Kim JW, Kim DK, Min A, Lee KH, Nam HJ, Kim JH, Kim JS, Kim TY, Im SA and Park IA: Amphiregulin confers trastuzumab resistance via AKT and ERK activation in HER2-positive breast cancer. J Cancer Res Clin Oncol 142(1): 157-65, 2016. PMID: 26195282. DOI: 10.1007/s00432-015-2012-4

26 Wang J, Zhu M, Wang L, Chen C and Song Y: Amphiregulin potentiates airway inflammation and mucus hypersecretion induced by urban particulate matter via the EGFR-PI3K $\alpha$ AKT/ERK pathway. Cell Signal 53: 122-131, 2019. PMID: 30291869. DOI: 10.1016/j.cellsig.2018.10.002

27 Kopp J, Wang GY, Kulmburg P, Schultze-Mosgau S, Huan JN, Ying K, Seyhan H, Jeschke MD, Kneser U, Bach AD, Ge SD Dooley S and Horch RE: Accelerated wound healing by in vivo application of keratinocytes overexpressing KGF. Mol Ther 10(1): 86-96, 2004. PMID: 15233945. DOI: 10.1016/j.ymthe. 2004.04.016

28 auf demKeller U, Krampert M, Kümin A, Braun S and Werner S: Keratinocyte growth factor: effects on keratinocytes and mechanisms of action. Eur J Cell Biol 83(11-12): 607-12, 2004. PMID: 15679105.

29 Gomperts BN, Belperio JA, Fishbein MC, Keane MP, Burdick MD and Strieter RM: Keratinocyte growth factor improves repair in the injured tracheal epithelium. Am J Respir Cell Mol Biol 37(1): 4856, 2007. PMID: 17332441. DOI: 10.1165/rcmb.2006-0384OC

30 Numanoğlu KV, Tatli D, Bektaş S and Er E: Efficacy of keratinocyte growth factor (palifermin) for the treatment of caustic esophageal burns. Exp Ther Med 8(4): 1087-1091, 2014. PMID: 25187801. DOI: 10.3892/etm.2014.1851

31 Dial CF, Tune MK, Doerschuk CM and Mock JR: Foxp3+ Regulatory T Cell Expression of Keratinocyte Growth Factor Enhances Lung Epithelial Proliferation. Am J Respir Cell Mol Biol 57(2): 162-173, 2017. PMID: 28296468. DOI: 10.1165/ rcmb.2017-0019OC

32 Yamamoto-Fukuda T, Akiyama N, Takahashi M and Kojima H: Keratinocyte Growth Factor (KGF) Modulates Epidermal Progenitor Cell Kinetics through Activation of p63 in Middle Ear Cholesteatoma. J Assoc Res Otolaryngol 19(3): 223-241, 2018. PMID: 29549594. DOI: 10.1007/s10162-018-0662-z

33 Hom YK, Young P, Thomson AA and Cunha GR: Keratinocyte growth factor injected into female mouse neonates stimulates uterine and vaginal epithelial growth. Endocrinology 139(9): 3772-9, 1998. PMID: 9724029. DOI: 10.1210/endo.139.9.6182
34 Masui F, Matsuda M and Mori T: Involvement of keratinocyte growth factor (KGF)-KGF receptor signaling in developmental estrogenization syndrome of mouse vagina. Cell Tissue Res 318(3): 591-8, 2004. PMID: 15480797. DOI: 10.1007/s00441-004-0980-9

35 Ceccarelli S, D’Amici S, Vescarelli E, Coluccio P, Matricardi P, di Gioia C, Benedetti Panici P, Romano F, Frati L, Angeloni A and Marchese $\mathrm{C}$ : Topical KGF treatment as a therapeutic strategy for vaginal atrophy in a model of ovariectomized mice. J Cell Mol Med 18(9): 1895-907, 2014. PMID: 25088572. DOI: $10.1111 / \mathrm{jcmm} .12334$

36 Zhang X, Ibrahimi OA, Olsen SK, Umemori H, Mohammadi M and Ornitz DM: Receptor specificity of the fibroblast growth factor family. The complete mammalian FGF family. J Biol Chem 281(23): 15694-700, 2006. PMID: 16597617. DOI: 10.1074/jbc.M601252200

37 Zinkle A and Mohammadi M: Structural Biology of the FGF7 Subfamily. Front Genet 10: 102, 2019. PMID: 30809251. DOI: 10.3389/fgene. 2019.00102

38 Grose R, Fantl V, Werner S, Chioni AM, Jarosz M, Rudling R, Cross B, Hart IR and Dickson C: The role of fibroblast growth factor receptor $2 \mathrm{~b}$ in skin homeostasis and cancer development. EMBO J 26(5): 1268-78, 2007. PMID: 17304214. DOI: 10.1038/sj.emboj.7601583

39 Yang J, Meyer M, Müller AK, Böhm F, Grose R, Dauwalder T, Verrey F, Kopf M, Partanen J, Bloch W, Ornitz DM and Werner S: Fibroblast growth factor receptors 1 and 2 in keratinocytes control the epidermal barrier and cutaneous homeostasis. J Cell Biol 188(6): 935-52, 2010. PMID: 20308431. DOI: 10.1083/ jcb.200910126

40 Meyer M, Müller AK, Yang J, Moik D, Ponzio G, Ornitz DM, Grose R and Werner S: FGF receptors 1 and 2 are key regulators of keratinocyte migration in vitro and in wounded skin. J Cell Sci 125(Pt 23): 5690-701, 2012. PMID: 22992463. DOI: $10.1242 /$ jcs. 108167

$41 \mathrm{Li} \mathrm{Q}$, Kannan A, DeMayo FJ, Lydon JP, Cooke PS, Yamagishi H, Srivastava D, Bagchi MK and Bagchi IC: The antiproliferative action of progesterone in uterine epithelium is mediated by Hand2. Science 331(6019): 912-6, 2011. PMID: 21330545. DOI: $10.1126 /$ science. 1197454

42 Nallasamy S, Li Q, Bagchi MK and Bagchi IC: Msx homeobox genes critically regulate embryo implantation by controlling paracrine signaling between uterine stroma and epithelium. PLoS Genet 8(2): e1002500, 2012. PMID: 22383889. DOI: 10.1371/journal.pgen.1002500

$43 \mathrm{Li}$ Y, Dowbenko D and Lasky LA: AKT/PKB phosphorylation of $\mathrm{p} 21 \mathrm{Cip} / \mathrm{WAF} 1$ enhances protein stability of p21Cip/WAF1 and promotes cell survival. J Biol Chem 277(13): 11352-61, 2002. PMID: 11756412. DOI: 10.1074/jbc.M109062200

44 Nakamura T, Miyagawa S, Katsu Y, Sato T, Iguchi T and Ohta Y: Sequential changes in the expression of Wnt- and Notchrelated genes in the vagina and uterus of ovariectomized mice after estrogen exposure. In Vivo 26(6): 899-906, 2012. PMID: 23160670 .

Received May 26, 2019

Revised June 30, 2019

Accepted July 3, 2019 